\title{
A Study on the Effectiveness of Turbo Equalization with FEC for Nonlinearity Compensatin in Coherent WDM Transmissions
}

\author{
Fujimori, T.,; Koike-Akino, T.; Sugihara, T.; Kubo, K.; Koguchi, K.; Mizuochi, T.; Ohshima, \\ C.; Nakashima, H.; Hoshida, T.
}

TR2013-053 June 2013

\begin{abstract}
We evaluate the performance improvement in the presence of fiber non- linearity obtained using a Turbo equalizer. Numerical simulation shows that Turbo equalization offers an improvement of $0.8 \mathrm{~dB}$ in a $100 \mathrm{~Gb} / \mathrm{s}$ NZ-DSF transmission, even with only 5 taps for the MAP estimation.

Conference on Lasers and Electro-Optics Pacific Rim and OptoElectronics and Communications Conference / Photonics in Switching (CLEO-PR \& OECC/PS)
\end{abstract}

This work may not be copied or reproduced in whole or in part for any commercial purpose. Permission to copy in whole or in part without payment of fee is granted for nonprofit educational and research purposes provided that all such whole or partial copies include the following: a notice that such copying is by permission of Mitsubishi Electric Research Laboratories, Inc.; an acknowledgment of the authors and individual contributions to the work; and all applicable portions of the copyright notice. Copying, reproduction, or republishing for any other purpose shall require a license with payment of fee to Mitsubishi Electric Research Laboratories, Inc. All rights reserved. 



\title{
A Study on the Effectiveness of Turbo Equalization with FEC for Nonlinearity Compensation in Coherent WDM Transmissions
}

\author{
Takafumi Fujimori*, Toshiaki Koike-Akino**, Takashi Sugihara*, \\ Kazuo Kubo*, Kazuumi Koguchi*, Takashi Mizuochi*, \\ Chihiro Ohshima***, Hisao Nakashima****, Takeshi Hoshida**** \\ * Information Technology R\&D Center, Mitsubishi Electric Corporation, 5-1-1, Ofuna, Kamakura, 247-8501, Japan \\ ** Mitsubishi Electric Research Laboratories, 201 Broadway, Cambridge, Massachusetts 02139, USA \\ *** Fujitsu Laboratories Ltd., 4-1-1, Kamikodanaka, Nakahara-ku, Kawasaki, 211-8588, Japan \\ **** Fujitsu Limited, 4-1-1, Kamikodanaka, Nakahara-ku, Kawasaki, 211-8588, Japan
}

\begin{abstract}
We evaluate the performance improvement in the presence of fiber nonlinearity obtained using a Turbo equalizer. Numerical simulation shows that Turbo equalization offers an improvement of $0.8 \mathrm{~dB}$ in a $100 \mathrm{~Gb} / \mathrm{s}$ NZ-DSF transmission, even with only 5 taps for the MAP estimation.
\end{abstract}

\section{INTRODUCTION}

Fiber nonlinearity has become a major limiting factor in ultra-high-speed optical communications, and numerous research efforts have been dedicated to mitigating nonlinear impairments. For example, digital back-propagation (DBP) offers a substantial advantage for nonlinear compensation [1, 2].

As an alternative, a maximum a posteriori probability (MAP) detector consisting of a sliding window was proposed [3]. We improved this by using high-order statistics [4]. These statistical equalizers achieve good performance, especially when combined with other methods, including a frequency-domain equalizer (FDE) to shorten the channel memory [5].

To improve the performance, Turbo equalization methods have been proposed for optical communications [6-8]. A "Turbo loop" is formed between a MAP equalizer and a soft-input soft-output (SISO) decoder that exchanges belief messages. We proposed a modified Turbo equalizer, which uses a sliding window MAP estimator, a low-density parity-check (LDPC) decoder, and a high-order statistical analyzer [8]. This achieved 2$4 \mathrm{~dB}$ gain for a single channel transmission line.

In this paper, we investigate in detail the performance improvement offered by Turbo equalization for three types of fiber link with WDM transmission, taking a realistic number of taps.

\section{Turbo EquALIZATION IN WDM SYSTEMS}

Fig. 1 shows the WDM coherent optical transmission system under consideration. LDPC-coded random data is generated at the coherent transmitter. We use perturbation pre-distortion. Five independent channels are multiplexed at $50 \mathrm{GHz}$ spacing. The WDM signals propagate through

This work was in part supported by the Lambda Reach project of the National Institute of Information and Communications Technology (NICT), as part of a program of the Ministry of Internal Affairs and Communications (MIC) of Japan. three types of fiber whose parameters are listed in Table 1. We insert additive white Gaussian noise (AWGN) at both ends of the fiber link to emulate the amplified spontaneous emission (ASE) noise of optical amplifiers. Here, we use identical power at both ends such that the total optical signal-to-noise power ratio (OSNR) is $15 \mathrm{~dB}$.

After the WDM de-multiplexer, the received signal of interest at the center channel is processed by the Turbo equalizer proposed in [8]. The received data is preprocessed by a series of units which include timing recovery, FDE for chromatic dispersion compensation, polarization mode dispersion (PMD) compensation, and DBP nonlinear compensation.

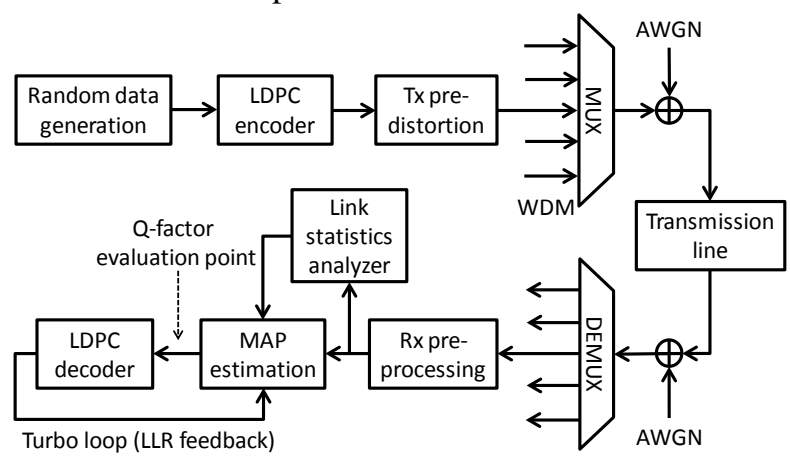

Fig. 1. Turbo equalization in a WDM optical transmission system.

Using training sequences, the pre-processed signals are also analyzed to obtain the high-order statistics of the residual nonlinearity. The MAP estimator generates the log-likelihood ratio (LLR) for the decoder by exploiting these statistics to compensate any data-pattern-dependent distortion. To improve the performance, the LLR extrinsic information is fed back from the decoder to the MAP estimator. We evaluate the Q-factor by counting the bit errors at the input of the LDPC decoder.

\section{SimUlation MODEL}

As shown in Table 1, we consider three different fiber transmission models. Case 1 is the longest at $2500 \mathrm{~km}$, and has no dispersion management. The lines for Cases 2 and 3 are just $1000 \mathrm{~km}$ long. We assume well-managed dispersion in a single-mode fiber (SMF) for Case 2, whereas for Case 3 no dispersion management is applied to the non-zero dispersion-shifted fiber (NZ-DSF). We expect the non-linear optical effects to be greater in Case 3 than Case 1. 
To deal with cycle slip issues, we use differential encoding for the quadrature phase-shift keying (QPSK). The number of taps for MAP estimation is 3 or 5 symbols. We evaluate the Q-factor performance by comparing the conventional coherent detection with differential decoding (1 tap, no Turbo loop). To reveal the potential of the Turbo loop, we assume perfect LLR feedback.

TABLE 1. Transmission line models

\begin{tabular}{|c|c|c|c|}
\hline Parameter & Case 1 & Case 2 & Case 3 \\
\hline Fiber type & \multicolumn{2}{|c|}{ SMF } & NZ-DSF \\
\hline Dispersion map & $\begin{array}{c}0 \% \\
\text { compensation } \\
\end{array}$ & $\begin{array}{c}\text { 95\% in-line } \\
\text { compensation }\end{array}$ & $\begin{array}{c}0 \% \\
\text { compensation }\end{array}$ \\
\hline $\begin{array}{l}\text { Dispersion } \\
\text { coefficient }\end{array}$ & \multicolumn{2}{|c|}{$\begin{array}{c}16.8 \\
\mathrm{ps} / \mathrm{nm} / \mathrm{km} \\
\end{array}$} & $\begin{array}{c}42.4 \\
\mathrm{ps} / \mathrm{nm} / \mathrm{km} \\
\end{array}$ \\
\hline $\begin{array}{l}\text { Dispersion slope } \\
\text { coefficient }\end{array}$ & \multicolumn{2}{|c|}{$\begin{array}{c}0.057 \\
\mathrm{ps} / \mathrm{nm}^{2} / \mathrm{km}\end{array}$} & $\begin{array}{c}0.045 \\
\mathrm{ps} / \mathrm{nm}^{2} / \mathrm{km}\end{array}$ \\
\hline $\begin{array}{c}\text { Nonlinearity } \\
\text { index }\end{array}$ & \multicolumn{2}{|c|}{$\begin{array}{c}27 \times 10^{-27} \\
\mathrm{~m}^{2} / \mathrm{W}\end{array}$} & $\begin{array}{c}24.8 \times 10^{-21} \\
\mathrm{~m}^{2} / \mathrm{W}\end{array}$ \\
\hline Effective area & \multicolumn{2}{|c|}{$86 \times 10^{-12} \mathrm{~m}^{2}$} & $55 \times 10^{-12} \mathrm{~m}^{2}$ \\
\hline Span length & \multicolumn{3}{|c|}{$100 \mathrm{~km} / \mathrm{span}$} \\
\hline Span loss & \multicolumn{3}{|c|}{$20 \mathrm{~dB} / \mathrm{span}$} \\
\hline Number of spans & $25(2500 \mathrm{k}$ & & $(1000 \mathrm{~km})$ \\
\hline Launched power & \multicolumn{3}{|c|}{-2 to $+4 \mathrm{dBm} / \mathrm{ch}$} \\
\hline ROADM filter & \multicolumn{3}{|c|}{$45 \mathrm{GHz}$, 4th order Gaussian } \\
\hline Channels & \multicolumn{3}{|c|}{5 wavelengths, $50 \mathrm{GHz}$ spacing } \\
\hline Received OSNR & \multicolumn{3}{|c|}{$15 \mathrm{~dB} / 0.1 \mathrm{~nm}$} \\
\hline LDPC overhead & \multicolumn{3}{|c|}{$20.5 \%$} \\
\hline
\end{tabular}

\section{Simulation RESUlts}

Figs. 2, 3 and 4 show the simulated Q-factor as a function of the fiber input power for the link model cases 1, 2, and 3. The Q-factor degrades with increased power because of the greater nonlinearity. The 3-tap Turbo equalizer offers only a slight performance gain compared to conventional coherent detection with differential decoding. On the other hand, it is seen that the performance degradation can be mitigated by Turbo equalization with 5 taps, especially in higher power regimes. From this, we can expect a significant improvement in a non-linear environment even with only 5-tap Turbo equalization.

Table 2 summarizes the performance improvement achieved. We can see that the turbo loop with 5 taps achieves $0.8 \mathrm{~dB}$ improvement in $100 \mathrm{~Gb} / \mathrm{s} \mathrm{NZ}-\mathrm{DSF}$ transmission. Although the conditions in the transmission paths are different, Turbo equalization gave a similar performance improvement in each case.

The reason why the improvements are not as great as expected is that a large part of the distortion is already equalized by the DBP before presentation to the Turbo equalizer. Moreover, the reason why the gain is lower than that for the single channel link reported in $[4,8]$ is that inter-channel nonlinearity effects dominate intrachannel nonlinearity effects in WDM transmissions.

\section{Conclusions}

We investigated Turbo equalization for nonlinearity compensation in WDM systems. It achieved about 0.8dB improvement using only 5-taps Turbo equalization, a realistic number of taps. The performance would be improved further by using more taps, which will be enabled by advance in LSI technology.

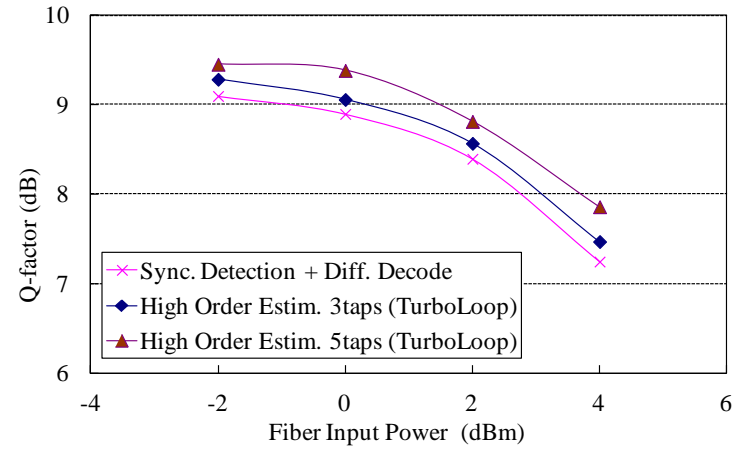

Fig. 2. Q-factor in Case 1.

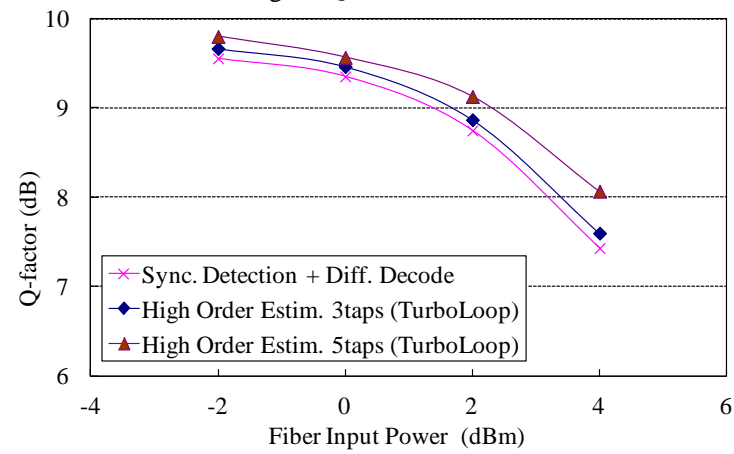

Fig. 3. Q-factor in Case 2.

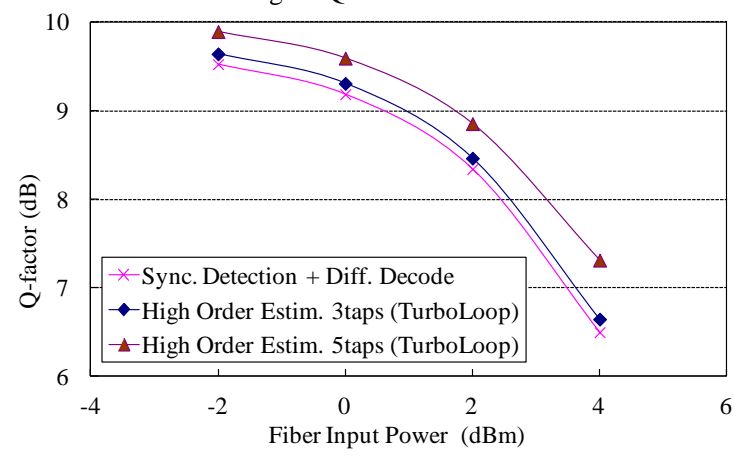

Fig. 4. Q-factor in Case 3.

TABLE 2. Performance improvements

\begin{tabular}{|c|c|c|c|c|c|c|}
\hline \multirow{2}{*}{$\begin{array}{c}\text { Input } \\
\text { Power } \\
{[\mathrm{dBm}]}\end{array}$} & \multicolumn{6}{|c|}{ Improvement in Q-factor [dB] } \\
\cline { 2 - 7 } & 3 taps & 5 taps & 3 taps & 5 taps & 3 taps & 5 taps \\
\hline-2 & 0.2 & 0.4 & 0.1 & 0.2 & 0.1 & 0.4 \\
\hline 0 & 0.2 & 0.5 & 0.1 & 0.2 & 0.1 & 0.4 \\
\hline 2 & 0.2 & 0.4 & 0.1 & 0.4 & 0.1 & 0.5 \\
\hline 4 & 0.2 & 0.6 & 0.2 & 0.6 & 0.1 & 0.8 \\
\hline
\end{tabular}

\section{REFERENCES}

[1] X. Li, et al., Opt. Express, vol. 16, no. 2, pp. 880-888, 2008.

[2] E. Ip, et al., IEEE J. Lightwave Technol., vol. 26, no. 20, pp. 3416-3425, 2008.

[3] Y. Cai, et al., OFC, OTuE1, 2010.

[4] T. Koike-Akino, et al., Opt. Express, vol. 20, no. 14, pp. 15769-15780, 2012.

[5] H. G. Batshon, et al., IEEE Photon. Journal, vol. 2, no. 4, pp. 593-599, Aug. 2010.

[6] I. B. Djordjevic, et al., IEEE JSAC, vol. 26, no. 6, pp. 7383, 2008.

[7] I. B. Djordjevic, et al., IEEE/OSA J. Opt. Commun. Netw., vol. 1, no. 6, pp. 555-564, Nov. 2009.

[8] C. Duan, et al., OFC, JW2A.5, 2012. 